\title{
When a nephrology ward becomes a COVID-19 ward: the Cremona experience
}

\author{
Fabio Malberti ${ }^{1} \cdot$ Paola Pecchini $^{1} \cdot$ Gianluca Marchi ${ }^{1} \cdot$ Marina Foramitti $^{1}$
}

Published online: 19 May 2020

(C) Italian Society of Nephrology 2020

Coronavirus disease 2019 (COVID-19), caused by the novel severe acute respiratory syndrome coronavirus 2 (SARSCoV-2), spread in Italy since February 2020 when the first case of COVID-19 was diagnosed in Codogno, a city in Lombardy, Northern Italy, about $20 \mathrm{~km}$ from Cremona. Since that time, Lombardy has become the epicentre of COVID-19 in Italy, with approximately $38 \%$ of the 165,155 confirmed COVID-19 cases recorded in Italy and $52 \%$ of the 21.645 reported COVID-19 deaths, as of April 15th, 2020 (www.salute.gov.it). The China Centre for Disease Control has recently published the largest COVID-19 series, which includes 44,672 cases. Beside age, the main risk factors were the presence of cardiovascular diseases, diabetes, chronic respiratory diseases, hypertension, and cancer; the mortality rate was $2.3 \%[1,2]$. However, in Italy in general, and in the Lombardy region in particular, mortality rates are much higher than in China [3]. Similar figures are being reported in other European countries, including France and Spain.

While patients with chronic kidney disease (CKD) are undoubtedly at high risk of developing a severe infection, data regarding the management and prognosis of CKD and renal transplant patients with COVID-19 are limited (see supplemental references, 1-6).

On this background we would like to report on our experience with the recent epidemic, when the renal ward in our hospital in Cremona served mainly for treatment of patients affected by COVID-19. From February 20 to April 15, 2020, a total of 2301 patients with severe or complicated SARS$\mathrm{CoV}-2$ infection were referred to the Emergency Department of Cremona Hospital. To face this emergency, the hospital

Electronic supplementary material The online version of this article (https://doi.org/10.1007/s40620-020-00743-y) contains supplementary material, which is available to authorized users.

Fabio Malberti

f.malberti@asst-cremona.it

1 Divisione Di Nefrologia E Dialisi, Renal Department, ASST-Cremona, Largo Priori 1, 26100 Cremona, Italy wards were re-organized to the detriment of a strict distinction between medical specialities. Thus, also patients without kidney disease were hospitalized in the Renal Department: overall, 82 cases of COVID-19 were managed, including 11 renal transplant recipients, 37 patients with CKD stage 3-5 or on dialysis at referral, and 34 patients with normal renal function. All patients had bilateral interstitial pneumonia documented by chest-computerised tomography (CT) and tested positive for SARS- CoV-2 by a throat swab sample using reverse transcription-polymerase chain reaction (RT-PCR).

Patients admitted to the Nephrology ward were older (median 74 years, IQ 61-81) than other hospitalized patients (n 1395, median 70 years, IQ 58-79); among them kidney transplant (KT) recipients were the youngest and CKD patients the oldest (Table 1). Only 4 of the $11 \mathrm{KT}$ recipients had an eGFR $>60 \mathrm{ml} / \mathrm{min} / 1.73 \mathrm{~m}^{2}$. Overall, $54.9 \%$ of the patients had at least one comorbidity (diabetes, ischemic heart disease, protein energy wasting, systemic disease, or active neoplasia) other than nephropathy. In keeping with the severity of the patients hospitalised under the pressure of the COVID-19 wave, $97.6 \%$ of the patients needed oxygen support (oxygen saturation $<93 \%$ and/or respiratory rate $>30$ breath/min); 35 patients (42.7\%) needed non-invasive ventilation (Table 1). Six patients (7.3\%) were transferred to the intensive care unit (ICU), and required mechanical ventilation. Four of them died in ICU.

Complications were extremely frequent in this negatively selected population, and involved $41.5 \%$ of patients. Acute renal failure (AKI), defined according to the KDIGO criteria, and sepsis were the most frequent complications (Table 1). Excluding the 20 patients on dialysis, 25 (40.3\%) of the 62 patients hospitalized in our ward developed AKI, in 6 severe enough to require dialysis. AKI was caused by renal hypoperfusion due to dehydration and/or hypotension (13 cases), drug-induced nephrotoxicity (high dose fluoroquinolones prescribed before hospitalization) (two cases), sepsis or septic shock (seven cases) and was associated to 
Table 1 Clinical features, respiratory support, and treatments of patients with COVID-19 interstitial pneumonia

\begin{tabular}{|c|c|c|c|c|c|}
\hline & All & $\begin{array}{l}\text { Normal kidney } \\
\text { function }\end{array}$ & CKD 3-5 & Hemodialysis (HD) & $\begin{array}{l}\text { Kidney trans- } \\
\text { plantation } \\
(\mathrm{KT})\end{array}$ \\
\hline Number & 82 & 34 & 17 & 20 & 11 \\
\hline Age, years & $70.5 \pm 15$ & $69.6 \pm 17$ & $78.7 \pm 9$ & $71.7 \pm 10$ & $58.5 \pm 11$ \\
\hline Males: n (\%) & $55(63.2)$ & $24(70.5)$ & $10(58.8)$ & $11(55.0)$ & $10(90.9)$ \\
\hline Diabetes: n (\%) & $28(34.1)$ & $8(23.5)$ & $10(58.8)$ & $9(45.0)$ & $1(9.1)$ \\
\hline CVD *: n $(\%)$ & $13(15.9)$ & $3(8.8)$ & $4(23.5)$ & $6(30.0)$ & 0 \\
\hline Active neoplasia: n (\%) & $3(3.7)$ & $2(5.9)$ & 0 & $1(5)$ & 0 \\
\hline \multicolumn{6}{|l|}{ Respiratory support: } \\
\hline Oxygen mask: n (\%) & $45(54.9)$ & $24(70.6)$ & $8(47.0)$ & $8(40.0)$ & $5(45.5)$ \\
\hline Noninvasive ventilation: $\mathrm{n}(\%)$ & $35(42.7)$ & $10(29.4)$ & $9(53.0)$ & $12(60.0)$ & $4(36.4)$ \\
\hline \multicolumn{6}{|l|}{ Anti-COVID-19 treatment: } \\
\hline Hydroxychloroquine \pm Azitromycin: $\mathrm{n}(\%)$ & $36(43.9)$ & $21(61.8)$ & $6(35.3)$ & $6(30.0)$ & $3(27.3)$ \\
\hline Lopinavir/Ritonavir: n (\%) & $13(15.8)$ & $6(17.6)$ & $5(29.4)$ & 0 & $2(18.2)$ \\
\hline Remdesivir: $\mathrm{n}(\%)$ & $1(1.2)$ & 0 & 0 & 0 & $1(9.1)$ \\
\hline Glucocorticoids: n (\%) & $31(37.8)$ & $11(32.4)$ & $4(23.5)$ & $6(30.0)$ & $11(100) \&$ \\
\hline Tocilizumab/Canakinumab: n (\%) & $7(8.5)$ & $6(17.6)$ & 0 & $1(5.0)$ & 0 \\
\hline Support therapy only & $31(37.8)$ & $12(35.3)$ & $10(58.8)$ & $9(45.0)$ & 0 \\
\hline \multicolumn{6}{|l|}{ Complications: } \\
\hline AKI: n (\%) & $25 / 62(40.3)$ & $12(35.2)$ & $8(47.0)$ & Excluded & $5(45.4)$ \\
\hline Dialysis for AKI: n (\%) & $6 / 62(9.7)$ & $3(8.8)$ & $1(5.9)$ & Excluded & $2(18.2)$ \\
\hline Sepsis: n (\%) & $11(13.4)$ & $4(11.7)$ & $2(11.8)$ & $2(10.0)$ & $3(27.3)$ \\
\hline $\mathrm{MI} / \mathrm{HF}^{* *}: \mathrm{n}(\%)$ & $5(6.1)$ & $2(5.9)$ & $2(11.8)$ & $1(5)$ & 0 \\
\hline Stroke: n (\%) & $1(1.2)$ & 0 & 0 & $1(5)$ & 0 \\
\hline Mechanical ventilation: $\mathrm{n}(\%)$ & $6(7.3)$ & $2(5.9)$ & $1(5.9)$ & 0 & $3(27.3)$ \\
\hline \multicolumn{6}{|l|}{ Outcome: } \\
\hline Died: $\mathrm{n}(\%)$ & $37(45.1)$ & $12(35.3)$ & $15(88.2)$ & $6(30.0)$ & $4(36.4)$ \\
\hline Discharged: n (\%) & $44(53.6) * * *$ & $21(61.7)$ & $2(12.8)$ & $14(65.0)$ & $7(63.6)$ \\
\hline
\end{tabular}

CKD 3-5 group included 7 cases with CKD stage 3, 6 with CKD stage 4, and 4 with CKD stage 5

*CVD: cardiovascular disease, including ischemic cardiopathy, dilatative cardiomyopathy, and congestive heart failure

** MI/HF: myocardial infarction/ hearth failure

***Hospitalization ongoing, as of April 27: 1 patient with normal kidney function

\& $=$ Glucocorticoids were added in 2 cases (metilprednisolone $16 \mathrm{mg} / \mathrm{day}$ ), and the dosage was increased (from the usual low dose of metilprednisolone $2-4 \mathrm{mg} /$ day or prednisone $2.5-5 \mathrm{mg}$ /day) to $16 \mathrm{mg}$ or $25 \mathrm{mg}$ of prednisolone or prednisone, respectively, in 2 cases

myocardial infarction or heart failure in 3 cases. Overall, 11 patients $(13 \%)$ developed sepsis or septic shock $(E$. coli in 3, Staphylococcus aureus in 6, Streptococcus Viridans, and Enterococcus Faecalis in the remaining 2 cases). One patient had stroke as a presenting symptom. Six patients $(7.3 \%, 3$ with normal kidney function, and 3 with CKD 3-5) had impaired consciousness. Two patients developed myocardial infarction in the first $72 \mathrm{~h}$ from admission, and died of its consequences. The median length of hospitalization was 7 days (IQ: 4-15 days). The duration of hospitalization was less in the deceased (4.9 days, IQ 3-10) than in discharged (8.3 days, IQ 5-18). Of note, $25 \%$ of deceased patients died within $72 \mathrm{~h}$ of hospitalization.

The severity of the disease, often characterized by a rapid and unpredictable worsening of the lung picture up to respiratory failure, lead us to offer our patients the treatments that, even in the absence of clear evidence, had both a reasonable pathophysiological background and had been tested in pilot studies with some promising results (supplemental refences, 7-13). This therapeutic approach has been shared overall in the Lombardy region, and applied also to dialysis patients, with careful assessment of possible adverse reaction, interferences between drugs, and dose adjustment in relation to renal function and body weight $[4,5]$.

We choose therefore a pharmacological strategy in twophases [5]: in the early one (within 7-10 days from the onset of symptoms) we prescribed drugs targeted against the viral infection: hydroxychloroquine (plus azithromycin, following the French experiences), lopinavir/ritonavir, and remdesivir [5]. In the second disease phase (generally 
over 7-10 days from the symptom onset), in case of a progressive lung involvement with escalating needs of oxygen supplementation and respiratory support, we prescribed corticosteroids or anti-cytokine drugs (tocilizumab, $8 \mathrm{mg}$ / $\mathrm{kg}$ of body weight iv for 2 days, as a first choice, anakinra $100 \mathrm{mg}$ subq for 7 days, or canakinumab $150 \mathrm{mg}$ subq, when toculizumab was not available), after carefully excluding concomitant infections, and in presence of high levels of IL-6, C reactive protein, and D-dimer. All patients received prophylactic therapy with enoxaparin at a dosage adjusted for renal function and body weight. Treatment choices were the result of a continuous work-in-progress: in fact, some drugs, including lopinavir/ritonavir, remdesivir, and tocilizumab, were not always available due to the high number of COVID-19 patients: over 600 patients were hospitalized simultaneously in Cremona, and about 13,000 patients were hospitalised in Lombardy on April 15th 2020 (https://www. salute.gov.it/imgs/C_17_notizie_4482_0_file.pdf). Furthermore, lopinavir/ritonavir was no more prescribed after the publication of a randomized trial not supporting its use (supplemental reference, 10). Moreover, concomitant therapies and comorbidities were considered in drug choice and, for example, hydroxychloroquine (plus Azithromycin) was more frequently prescribed in patients with normal renal function, and without severe cardiac disease. All KT recipients discontinued antimetabolite therapy (11/11) and most of them calcineurin inhibitors (8/11). In all transplanted patients the corticosteroid dose was increased or started. Only about one third of the entire population (31 cases; $37.8 \%$ ) received only supportive therapy.

As of April 27, 37 patients (45.1\%) had died, and 44 patients (53.6\%) had been discharged from the hospital. Mortality was higher in our patients than in the remaining COVID-19 positive population hospitalized in the Cremona hospital in the same period (425 deaths in 1395 hospitalized patients, 30.5\%). Mortality was particularly high in CKD 3-5 (88.2\%) patients, who were also the oldest and sickest. Indeed, the effect of age may account for the higher mortality in CKD 3-5 than in dialysis or transplant patients. As expected, beside age $(75 \pm 10$ years vs $67 \pm 16$ years of discharged patients, $\mathrm{p}<0.001)$, patients who died had also a higher prevalence of complications, including AKI (43.2\% vs $20.0 \%$ ) or sepsis $(20.0 \%$ vs $8.9 \%)$. Mortality increased with age reaching $69 \%$ in the 29 patients older than 80 years, $55 \%$ in the 21 patients aged $70-79$ years, and $18.7 \%$ in the 32 patients under 70 years of age.

Within the limits of the selection bias and the treatment changes, when we tried to analyze the factors associated with in-hospital death (supplemental Table 1) with a multivariate logistic regression analysis, we confirmed that CKD 3-5 and age were significant predictors of mortality, whereas hydroxychloroquine therapy was associated with a lower risk, possibly also because this drug was prescribed in cases referred earlier to our ward. While data are too scattered to allow any sound conclusion, we would like to underline that, at least in our series, despite being older, HD patients had lower mortality than transplant recipients and patients with normal renal function, thus suggesting that HD patients could be relatively protected from violent cytokine storm by their impaired immune system (supplemental reference, 6). Death rate in our HD patients suffering from pneumonia was $30 \%$, slightly higher than that reported overall in the surveys on COVID-19 patients on dialysis in the Piedmont (25\%) and Lombardy (22\%) regions $[6,7]$.

The outcome of our KT recipients was in line with other reports [8] (supplemental reference, 1, 2), probably because we followed similar treatment strategies: we discontinued antimetabolite therapy in all the patients, calcineurin inhibitors in most of them, and increased or started steroids in all (Table 1).

What can our single center, stormy but still relatively limited experience, teach to a wider nephrology audience?

First, the need for nephrologists of being informed and trained to the care of COVID-19 patients, since they can face particular clinical challenges, both from the clinical and the organisational point of view. Even if we hope that the experience of transforming a renal ward into a Covid-19 renal ward will remain isolated, our role in managing CKD patients and $\mathrm{AKI}$ is undeniable.

Second, our experience, shared by many physicians who found themselves in the difficult battle against an unknown disease, shows how difficult it is to choose to treat or not to treat patients, on the basis of scattered experience and fragmentary data. The high mortality observed since the first days of the epidemic, prompted doctors to use therapies only on their pathophysiological basis for trying to interfere with disease progression. Most of the drugs have been used off label or on a compassionate-use basis, and often with unconvincing results. These clinical and ethical dilemmas show how the current evidence-based approach to treating disease may not be useful to deal with a rapid and violent epidemic, and request a reappraisal of our clinical decision pathway.

\section{Compliance with ethical standards}

Conflict of interest All the authors declare that they have no confictsof interest.

Ethical statement All procedures performed in studies involving human participants were in accordance with the ethical standards of the institutional and national research committee and with the 1964 Helsinki declaration and its later amendments or comparable ethical standards.

Informed consent For this type of study formal consent is not required. 


\section{References}

1. Huang C, Wang Y, Li X, Ren L, Zhao J, Hu Y et al (2020) Clinical features of patients infected with 2019 novel coronavirus in Wuhan, China. Lancet 395(10223):497-506

2. Grasselli G, Pesenti A, Cecconi M (2020) Critical care utilization for the COVID-19 outbreak in Lombardy, Italy: early experience and forecast during an emergency response. JAMA. https://doi. org/10.1001/jama.2020.4031

3. Onder G, Rezza G, Brusaferro S (2020) Case-fatality rate and characteristics of patients dying in relation to COVID-19 in Italy. JAMA. https://doi.org/10.1001/jama.2020.4683

4. Rombolà G, Heidempergher M, Pedrini L, Farina M, Aucella F, Messa P, Brunori G (2020) Practical indications for the prevention and management of SARS-CoV-2 in ambulatory dialysis patients: lessons from the first phase of the epidemics in Lombardy. J Nephrol 33:193-196

5. Alberici F, Delbarba E, Manenti C et al (2020) Management of patients on dialysis and with kidney transplant during SARSCOV-2 (COVID-19) pandemic in Brescia Italy. Kidney Int Rep. https://doi.org/10.1016/j.ekir.2020.04.001
6. Rombolà G, Brunini F (2020) COVID-19 and dialysis: why we should be worried. J Nephrol. https://doi.org/10.1007/s4062 0-020-00737-w

7. Manganaro M, Baldovino S, Working group of the Piedmont, and Aosta Valley Section of the SIN (2020) First considerations on the SARS-CoV-2 epidemic in the dialysis units of Piedmont and Aosta Valley Northern Italy. J Nephrol. https://doi.org/10.1007/ s40620-020-00732-1

8. Alberici F, Delbarba E, Manenti C et al (2020) A single center observational study of the clinical characteristics and shortterm outcome of 20 kidney transplant patients admitted for SARS-CoV2 pneumonia. Kidney Int. https://doi.org/10.1016/j. kint.2020.04.002

Publisher's Note Springer Nature remains neutral with regard to jurisdictional claims in published maps and institutional affiliations. 